\title{
APPLICATION OF THE COUPLED BEM/FEM METHOD FOR CALCULATION OF CATHODIC PROTECTION SYSTEM PARAMETERS
}

\author{
ADNAN MUJEZINOVIĆ ${ }^{1}$, SANJA MARTINEZ ${ }^{2}$, ALIJA MUHAREMOVIĆ ${ }^{1} \&$ IRFAN TURKOVIĆ $^{1}$ \\ ${ }^{1}$ University of Sarajevo, Faculty of Electrical Engineering, Bosnia \& Herzegovina. \\ ${ }^{2}$ University of Zagreb, Faculty of Chemical Engineering and Technology, Croatia.
}

\begin{abstract}
Cathodic protection $(\mathrm{CP})$ is a technique that prevents corrosion of underground metallic structures. Design of any CP system first requires defining the protection of current density and potential distribution, which should meet the given criterion. It also needs to provide, as uniform as possible, current density distribution on the protected object surface. Determination of current density and potential distribution of CP system is based on solving the Laplace partial differential equation. Mathematical model, along with the Laplace equation, is represented by two additional equations that define boundary conditions. These two equations are non-linear and they represent the polarization curves that define the relationship between current density and potential on electrode surfaces. Nowadays, the only reliable way to determine current density and potential distribution is by applying numerical techniques. This paper presents efficient numerical techniques for the calculation of current density and potential distribution of CP system based on the coupled boundary element method (BEM) and finite element method (FEM).
\end{abstract}

Keywords: Boundary Element/Finite Element Method (BEM/FEM), Cathodic Protection (CP), non-linear boundary conditions, potential distribution.

\section{INTRODUCTION}

Due to the increasing number of underground and underwater metallic installations (such as pipelines), interest in the development of appropriate protection against corrosion processes has been growing. Practice has shown that the most effective way to protect these installations against corrosion is to use cathodic protection (CP) systems along with passive protection (high resistive coatings). These systems are assumed to be effective when the rate of corrosion of the protected object does not exceed a defined value, after installation of the CP system [1]. For the assessment of the efficiency of such systems, both in the design phase and during execution, it is necessary to know the distribution of the electric potential and protection current density on the surface of the protected object. Nowadays, for design and assessment of the effectiveness of such systems, numerical methods are used [2-4].

One of the most commonly used numerical methods for the calculation of relevant parameters of the CP systems is the boundary element method (BEM). The advantage of this method compared to other conventional numerical methods such as the finite difference method (FDM) and finite element method (FEM) is in that it only requires a discretization of the boundary surfaces. In addition, with this method it is relatively easy to treat infinite and semi-infinite domains such as ground and air. The only disadvantage of BEM is that it considers the electric potential on the electrode surface as being uniform; that is, there is no current flow through the electrodes $[2,5]$. This problem can be overcome by combining BEM with e.g. FEM. 
When modelling a CP system, electrochemical reactions that take place on the electrode surfaces must be taken into account. Since the electrochemical reactions take place on the electrode surface, they take into account the boundary conditions. Electrochemical reactions that take place on the electrode surface do not give the linear relationship between electric potential and current density; therefore, used boundary conditions are non-linear. Therefore, Newton-Raphson technique was used to solve the non-linear equation system [6, 7].

In this paper, coupled BEM/FEM method was used for calculation of the CP system parameters. Electrochemical reactions that occur on the electrode surface are considered in the presented mathematical model. In addition, attenuation of electric potential in the cathodically protected pipeline was taken into account.

\section{MATHEMATICAL MODEL}

The presented mathematical model is composed of three parts. The first part is the soil (outer domain), which is modelled using direct BEM; the second part is electrodes metal (inner domain), which is modelled by FEM; and the third part is the boundary between these two domains and is modelled by BEM/FEM [8-10]. In the third part, non-linear boundary conditions were applied.

\subsection{Boundary element method}

As previously mentioned, BEM was used for calculation of the electric potential and current density distribution in the outer domain, i.e. soil. According to the direct BEM, current density and electric potential can be calculated at any point of the domain by using the following integral equation:

$$
c(q) \varphi_{s}(q)+\int_{\Gamma} \varphi_{s}(p)(\vec{n} \cdot \nabla G(p, q)) d \Gamma=\int_{\Gamma} G(p, q)\left(\vec{n} \cdot \nabla \varphi_{s}(p)\right) d \Gamma+\varphi^{\prime},
$$

where $q$ is the observation point, $p$ is the field source point, $c(q)$ is the constant, $\varphi_{s}(q)$ is the potential at the observation point $q$ in soil, $\varphi_{s}(p)$ is the potential at the field source point $p$ in soil, $\vec{n}$ is the normal unit vector, $G(p, q)$ is the Green's function, $\Gamma$ is the boundary of the domain and $\nabla$ is Nabla operator. Additional term $\varphi^{\prime}$ given on the right side of the eqn (1) is the constant potential on the infinite boundary $[6,7,11]$. To calculate this potential, additional integral equation must be added [5-7]:

$$
\int_{\Gamma} \kappa_{S}\left(\vec{n} \cdot \nabla \varphi_{s}(p)\right) d \Gamma=0
$$

where $\kappa_{S}$ represents the electric soil conductivity.

Green's function used in this paper is given by the following equation:

$$
G(p, q)=\frac{1}{4 \pi}\left(\frac{1}{|p-q|}+\frac{1}{\left|p^{\prime}-q\right|}\right)
$$

where $|p-q|$ represents the distance between source point $p$ and observation point $q$ and $\left|p^{\prime}-q\right|$ is the distance between observation point $q$ and image of the source point $p^{\prime}$. The reflection of the source point $p^{\prime}$ is caused by soil/air interface and this point is placed in air on the distance from the soil/air interface similar to the source point $p$ placed in soil from the soil/air interface [10]. 
From integral eqns (1) and (2), potential in the soil at the soil/electrode interface as well as the flux density can be obtained. To calculate the current density on the electrodes, Ohms law must be introduced, as follows:

$$
j_{S}=-\kappa_{S} \nabla \varphi_{S}
$$

where $j_{S}$ represents current density.

Equations (1) and (2) can be solved by using direct BEM [12]. The first step of BEM is discretization of electrode surfaces with boundary elements. In this paper, biquadratic boundary element with nine nodes was used. After application of the Collocation method at the point and Gauss-Legendre quadrature, solution of the electric potential and current density can be obtained for each node of all boundary elements. This can be done by solving the following matrix equation:

$$
\left[\begin{array}{ccc}
H_{c c} & H_{c a} & -1 \\
H_{a c} & H_{a a} & -1 \\
0 & 0 & 0
\end{array}\right] \cdot\left\{\begin{array}{c}
\varphi_{S, c} \\
\varphi_{S, a} \\
\varphi_{\infty}
\end{array}\right\}=\left[\begin{array}{cc}
G_{c c} & G_{c a} \\
G_{a c} & G_{a a} \\
A_{c} & A_{a}
\end{array}\right] \cdot\left\{\begin{array}{c}
j_{S, c} \\
j_{S, a}
\end{array}\right\}
$$

In eqn (5), indexes $c$ and $a$ represent the cathode and anode surface, respectively.

According to the direct BEM, elements of the matrix eqn (5) can be calculated by using the following equations:

$$
\begin{gathered}
H_{i, j}=\sum_{j_{1}=1}^{N G} \sum_{j_{2}=1}^{N G} \sum_{m=1}^{N t} N_{m}\left(\xi_{j, j_{1}}, \eta_{j, j 2}\right) \cdot\left(\vec{n} \cdot \nabla G_{k}\left(\xi_{j, j_{1}}, \eta_{j, j_{2}}, q_{i}\right)\right) \operatorname{det}\left[J\left(\xi_{j, j_{1}}, \eta_{j, j 2}\right)\right] \omega_{j_{1}} \omega_{j_{2}} \\
G_{i, j}=-\frac{1}{\kappa_{S}} \sum_{j_{1}=1}^{N G} \sum_{j_{2}=1}^{N G} \sum_{m=1}^{N t} N_{m}\left(\xi_{j, j_{1}}, \eta_{j, j 2}\right) \cdot G_{k}\left(\xi_{j, j_{1}}, \eta_{j, j_{2}}, q_{i}\right) \operatorname{det}\left[J\left(\xi_{j, j_{1}}, \eta_{j, j 2}\right)\right] \omega_{j_{1}} \omega_{j_{2}} \\
A_{j}=\sum_{j_{1}=1}^{N G} \sum_{j_{2}=1}^{N G} \sum_{m=1}^{N t} N_{m}\left(\xi_{j, j_{1}}, \eta_{j, j 2}\right) \cdot \operatorname{det}\left[J\left(\xi_{j, j_{1}}, \eta_{j, j 2}\right)\right] \omega_{j_{1}} \omega_{j_{2}}
\end{gathered}
$$

where $N G$ is the number of applied Gauss-Legendre quadratures, $N_{t}$ is the number of nodes of one boundary element, $\xi, \eta$ are Gauss points, $N(\xi, \eta)$ are shape functions, $\operatorname{det}[J(\xi, \eta)]$ is determinant of Jacobean matrix, $w_{j 1}$ and $w_{j 2}$ are weights coefficients for Gauss points $\xi$ and $\eta$, respectively.

Equations (6) and (7) are valid for non-singular elements i.e. for $i \neq j$. Singular $H$ elements are calculated according to the Gibbs theorem [2]. Singular $G$ elements are split into two parts. The first part is a non-singular part and represents the impact of the image of the source point on the collocation point and is calculated as per eqn (7). The second part is a singular part and this part is calculated by using 1/r singular quadrature [13].

\subsection{Finite element method}

For calculation of the attenuation of the potential in the electrodes, FEM can be applied. The first step of FEM is discretization of the entire domain of interest on finite elements. After discretization, solution of the electric potential distribution can be calculated by solving the following integral equation: 


$$
\sum_{e=1}^{n_{f e}}\left[\kappa_{M} \int_{\Omega_{e}} \nabla \psi_{i}^{(e)} \nabla \psi_{j}^{(e)} d \Omega_{e}\right] \cdot \varphi_{M j}^{(e)}=\sum_{e=1}^{n_{f e}}\left[\int_{\Gamma_{e}} \psi_{i}^{(e)} \psi_{j}^{(e)} d \Gamma_{e}\right] \cdot j_{M j}^{(e)}
$$

where $\kappa_{M}$ is the conductivity of electrodes materials, $\Omega_{\mathrm{e}}$ is the volume of e-th finite element, $\psi$ is the shape function, $n_{f e}$ is the total number of finite elements, $\Gamma_{e}$ is the boundary surface of e-th finite element, $\varphi_{M j}{ }^{(e)}$ is the potential of $j$-th node of e-th finite element and $j_{M j}{ }^{(e)}$ is the current density of $\mathrm{j}$-th node of e-th finite element. Indexes $i$ and $j$ with shape functions represent the node number of e-th finite element.

Solution of eqn (9) can be written in the matrix form as follows:

$$
\left[\begin{array}{cc}
K_{c c} & 0 \\
0 & K_{a a}
\end{array}\right] \cdot\left\{\begin{array}{l}
\varphi_{M, c} \\
\varphi_{M, a}
\end{array}\right\}=\left[\begin{array}{cc}
F_{c c} & 0 \\
0 & F_{a a}
\end{array}\right] \cdot\left\{\begin{array}{l}
j_{M, c} \\
j_{M, a}
\end{array}\right\}
$$

After application of the coordinate transformation and Gauss-Legendre quadrature, the elements of matrix eqn (10) can be calculated according to the following equations $[2,5]$ :

$$
\begin{gathered}
K_{i, j}=\kappa_{M} \sum_{j_{1}=1}^{N G} \sum_{j_{2}=1}^{N G} \sum_{j_{3}=1}^{N G}\left[\sum_{k} \frac{\partial \psi_{i}(\xi, \eta, \zeta)}{\partial x_{k}} \frac{\partial \psi_{j}(\xi, \eta, \zeta)}{\partial x_{k}}\right] \cdot \operatorname{det}[J(\xi, \eta, \zeta)] \cdot \omega_{j_{1}} \cdot \omega_{j_{2}} \cdot \omega_{j_{3}} \\
F_{i, j}=\sum_{j_{1}=1}^{N G} \sum_{j_{2}=1}^{N G}\left[\psi_{i}(\xi, \eta) \cdot \psi_{j}(\xi, \eta)\right] \cdot \operatorname{det}[J(\xi, \eta)] \cdot \omega_{j_{1}} \cdot \omega_{j_{2}}
\end{gathered}
$$

where $x_{k}$ represents one of the Cartesian coordinates.

Equations (11) and (12) are valid for matrix elements of one finite element. To form global matrix eqn (10), it is necessary to take into account contributions of all finite elements that share the same node. This can be easily done by summing the contributions of all finite elements of a given node.

\subsection{BEM/FEM and boundary conditions}

To couple FEM for the inner domain with direct BEM for the outer domain, an additional equation must be added in the system. On the interface between two domains, a continuity equation can be written:

$$
-\kappa_{S}\left(\vec{n} \cdot \nabla \varphi_{S}\right)=-\kappa_{M}\left(\vec{n} \cdot \nabla \varphi_{M}\right) \Rightarrow j_{S}=j_{M}
$$

After application of the continuity equation, the matrix equation of the whole system can be written in the following form [2]:

$$
\left[\begin{array}{ccccc}
H_{c c} & H_{c a} & 0 & 0 & -1 \\
H_{a c} & H_{a a} & 0 & 0 & -1 \\
0 & 0 & K_{c c} & 0 & 0 \\
0 & 0 & 0 & K_{a a} & 0 \\
0 & 0 & 0 & 0 & 0
\end{array}\right] \cdot\left\{\begin{array}{c}
\varphi_{S, c} \\
\varphi_{S, a} \\
\varphi_{M, c} \\
\varphi_{M, a} \\
\varphi_{\infty}
\end{array}\right\}=\left[\begin{array}{cc}
G_{c c} & G_{c a} \\
G_{a c} & G_{a a} \\
F_{c c} & 0 \\
0 & F_{a a} \\
A_{c} & A_{a}
\end{array}\right] \cdot\left\{\begin{array}{c}
j_{c} \\
j_{a}
\end{array}\right\}
$$


To solve matrix eqn (14) it is necessary to apply boundary conditions on the all boundary surfaces of the problem. Boundary conditions on electrodes surfaces are defined by expressions that represent the relationship between electric potential and current density. These relationships represent the polarization characteristics of electrodes. For protected object (cathode) surface, thw polarization characteristic is given by the following expressions:

$$
j_{c}=j_{0 F e} \cdot 10^{\frac{\varphi_{M}-\varphi_{S}-\varphi_{F e}}{\beta_{F e}}}-j_{\lim , O_{2}}-j_{0 H_{2}} \cdot 10^{\frac{-\left(\varphi_{M}-\varphi_{S}-\varphi_{H_{2}}\right)}{\beta_{H_{2}}}}
$$

where $j_{\mathrm{OFe}}$ is the current density corresponding to the metal dissolution reaction, $j_{l i m, \mathrm{O}_{2}}$ is the limiting current density of oxygen reduction, $j_{\mathrm{H}_{2}}$ is the current density of hydrogen separation, $\varphi_{\mathrm{Fe}}, \varphi_{\mathrm{O}_{2}}$ and $\varphi_{\mathrm{H}_{2}}$ are the equilibrium potentials of corresponding electrochemical reactions and $\beta_{\mathrm{Fe}}, \beta_{\mathrm{O}_{2}}$ and $\beta_{\mathrm{H}_{2}}$ are Tafel slopes of metal dissolution, oxygen reduction and hydrogen separation, respectively.

Polarization characteristic for galvanic anode is defined by the following expression:

$$
j_{a}=j_{O_{2}} \cdot\left(10^{\frac{\varphi_{M}-\varphi_{S}-\varphi_{a}}{\beta_{a}}}-1\right)
$$

where $\varphi_{a}$ is the equilibrium potential of galvanic anode and $\beta_{a}$ is the Tafel slope of galvanic anode.

Equations (15) and (16) represent the boundary conditions of electrode surfaces. As it can be noted, these boundary conditions are non-linear and, therefore, matrix eqn (14) is nonlinear and can be solved by using iterative techniques. In this paper, for a solution of this equation the well-known Newton-Raphson iterative technique was used [12].

\section{CASE STUDY}

The previously presented mathematical model was used for the calculation of relevant parameters of the steel pipeline protected by galvanic anode CP system shown in Fig. 1.

Zinc galvanic anodes are placed alternately on both sides of the protected pipeline being spaced at $6 \mathrm{~m}$ and the distance from the pipeline axis is $5.5 \mathrm{~m}$. The outer diameter of the analyzed pipeline is $4 \mathrm{~m}$ and is set to a depth of $3.75 \mathrm{~m}$. The anode strings are placed at a

Galvanic anode

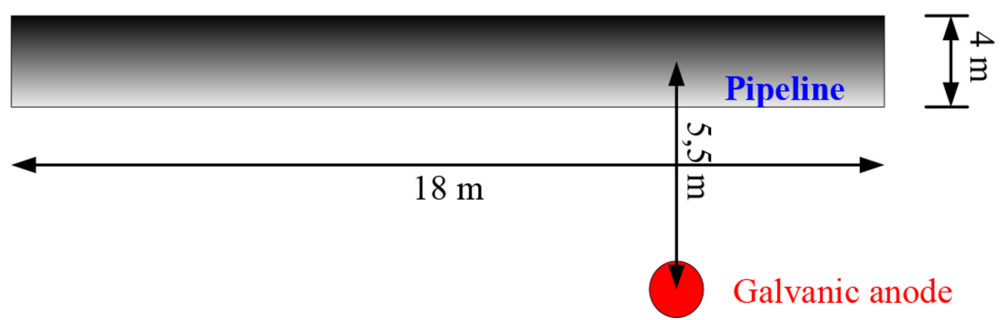

Figure 1: Geometry of analyzed system. 
depth of $2.5(\mathrm{~m})$, while the length of anode strings is $6.4 \mathrm{~m}$. The whole system is placed in the soil with electrical soil resistivity of $100 \Omega \mathrm{m}$. Value of polarization characteristics parameters for both, anode and cathode surfaces, are given in Table 1.

Calculation results of the electric potential and current density on the protected pipeline surface are given in Figs 2 and 3, respectively.

From results given on Figs 2 and 3, it can be noted that most of the negative values of the electric potential on the protected object are on the surfaces that are closer to the anode strings. With increase in distance from the anode string, the electrical potential of the protected object is closer to the values of corrosion potential (i.e. becomes more positive). The

Table 1: Value of polarization parameters $[2,11]$.

\begin{tabular}{llll}
\hline Parameter & Value & Parameter & Value \\
\hline$j_{\mathrm{OFe}}$ & $1\left(\mu \mathrm{A} / \mathrm{cm}^{2}\right)$ & $\varphi_{\mathrm{H}_{2}}$ & $-800(\mathrm{mV}) \mathrm{vs.} \mathrm{CSE}$ \\
$\varphi_{\mathrm{Fe}}$ & $-700(\mathrm{mV}) \mathrm{vs.} \mathrm{CSE}$ & $\beta_{\mathrm{H}_{2}}$ & $276,3(\mathrm{mV} / \mathrm{dec})$ \\
$\beta_{\mathrm{Fe}}$ & $138,15(\mathrm{mV} / \mathrm{dec})$ & $j_{O_{2}}$ & $1\left(\mu \mathrm{A} / \mathrm{cm}^{2}\right)$ \\
$j_{\text {limO }}$ & $1,1\left(\mu \mathrm{A} / \mathrm{cm}^{2}\right)$ & $\varphi_{a}$ & $-1100(\mathrm{mV}) \mathrm{vs.} \mathrm{CSE}$ \\
$j_{\mathrm{H}_{2}}$ & $1\left(\mu \mathrm{A} / \mathrm{cm}^{2}\right)$ & $\beta_{a}$ & $60(\mathrm{mV} / \mathrm{dec})$ \\
\hline
\end{tabular}

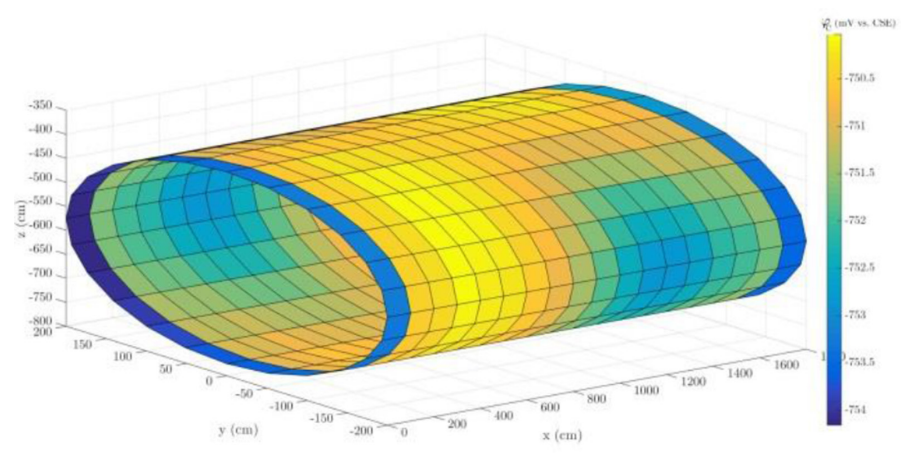

Figure 2: Electric potential distribution on pipeline surface.

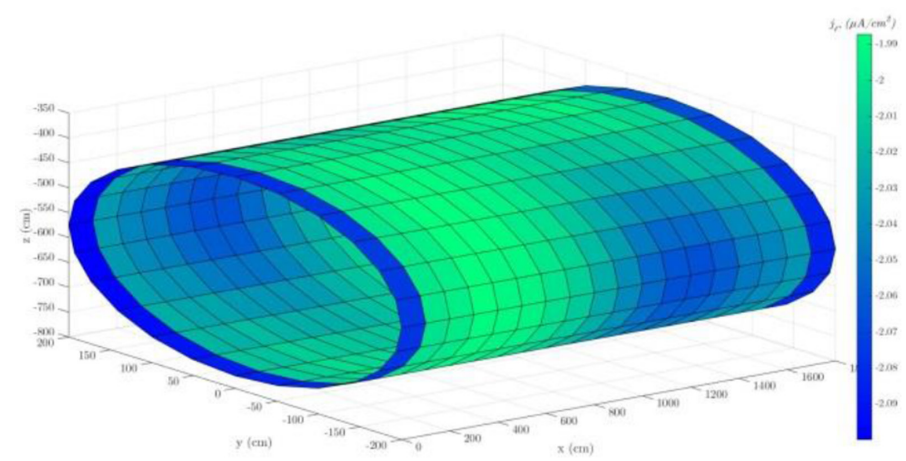

Figure 3: Current density distribution on pipeline surface. 
same applies for the distribution of current density. From given results it is also noticeable that electric potential and current density change the longitudinal as well as the axial on analyzed pipelines. In many analyses, axial change of the electric potential and current density are often neglected. From the given results it is clear that axial change of electric potential and current density is not negligible, and must be taken into consideration.

\section{CONCLUSION}

The main task in determining the efficiency of a CP system is to define technically the correct level of the electric potential and current density on the surface of the protected object. The most reliable way to adopt for calculation of these parameters is by the application of appropriate numerical methods. In this paper, a mathematical model based on the coupled BEM/ FEM for calculation of the electric potential and current density of the CP system is presented. In the presented model, direct BEM was used for the calculation of the electric potential and current density in the outer domain, and FEM for the calculation in the inner domain. These two methods are coupled on boundary between the outer and inner domains where the non-linear boundary conditions are applied.

The presented mathematical model was used for the calculation of electric potential and current density distribution of one illustrative example.

\section{ACKNOWLEDGEMENT}

This paper was supported by the Ministry of Education and Science of the Federation of Bosnia and Herzegovina.

\section{REFERENCES}

[1] Mujezinović, A., Martinez, S. \& Muharemović, A., Mathematical model for cathodic protection of the underground pipelines. 25th International Expert Meeting Komunalna Energetika/Power Engineering, 2016.

[2] Riemer, D.P., Modeling cathodic protection for pipeline networks, $\mathrm{PhD}$ Theses, University of Florida, USA, Florida, 2000.

[3] Adey, R.A. \& Hang, P.Y., Computer simulation as an aid to corrosion control and reduction. NACE Corrosion Conference, USA, Texas, San Antonio, 1999.

[4] Amaya, K., Mathematical modeling for corrosion analysis. Modelling of Cathodic Protection Systems, 12(1), pp. 1-12, 2005. http://dx.doi.org/10.2495/1-85312-889-9/01

[5] Riemer, D.P. \& Orazem, M.E., Modeling coating flaws with non-linear polarization curves for long pipelines. Corrosion and Boundary Element Methods, Advances in Boundary Elements, 12(1), pp. 225-259, 2005.

[6] Santiago, J.A.F. \& Telles, J.C.F., On boundary elements for simulation of cathodic protection system with dynamic polarization curves. International Journal for Numerical Methods in Engineering, 40(14), pp. 2611-2627, 1997.

http://dx.doi.org/10.1002/(SICI)1097-0207(19970730)40:14<2611::AID-NME178> 3.0.CO;2-P

[7] Santiago, J.A.F. \& Telles, J.C.F., A solution technique for cathodic protection system with dynamic boundary conditions by the boundary element method. Advances in Engineering Software, 30(9), pp. 663-671, 1999.

http://dx.doi.org/10.1016/S0965-9978(98)00121-5 
[8] Muharemovic, A., Zildzo, H., Behlilovic, N. \& Turkovic, I., Numerical model for calculation of parameters of cathodic protection system with galvanic anodes. XXII International Symposium on Information, Communication and Automation Technologies (ICAT), 2009.

[9] Muharemovic, A., Zildzo, H. \& Letic, E., Modelling of protective potentials distribution in cathodic protection systems using cupled BEM/FEM method. In 30th International conference on Boundary Elements Method and Other Reduction Methods, BEM/ MRM 30, Maribor, Slovenia, 2008. http://dx.doi.org/10.1109/icat.2009.5348399

[10] Muharemovic, A., Turkovic, I., Muharemovic, A., Tasakovic, S. \& Mujezinovic, A., Calculation methods of cathodic protection system parameters with vertical anodie zinc strings. 20th International Expert Meeting, Power Engineering, Maribor, Slovenia, 2011. http://dx.doi.org/10.2495/BE080111

[11] Martinez, S., Evaluation of the uniform current density assumption in cathodic protection systems with close anode-to-cathode arrangement. Materials and Corrosion, 61(4), pp. 338-342, 2010.

[12] Mujezinović, A., Turković, I., Martinez, S., Milojković, S., Modelling of the cathodic protection system with dynamic non-linear polarization characteristics. XXV International Conference on Information, Communication and Automation Technologies (ICAT), 2015. http://dx.doi.org/10.1109/icat.2015.7340536

[13] Brebbia, C.A., Telles, J.C.F. \& Wrobel, L.C., Boundary Element Techniques Theory and Applications in Engineering, Springer-Verlag: Berlin/Heidelberg, 1984. 